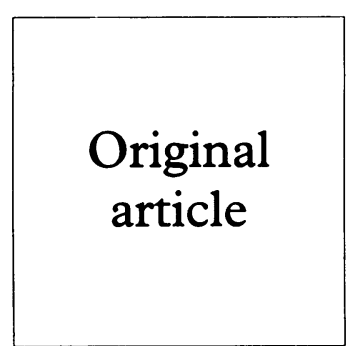

Sydney Hospital, Sydney, Australia N J Bodsworth

GlaxoWellcome

Research and

Development,

Beckenham, UK

R J Crooks

A J Ingamells

A Gibb

Dermatologische

Klinik und Poliklinik,

Munich, Germany

$S$ Borelli

Rigshospitalet,

Copenhagen, Denmark

$G$ Vejlsgaard

University Central

Hospital, Helsinki,

Finland

J Paavonen

Bispebjerg Hospital,

Copenhagen, Denmark

A-M Worm

Citymottagningen,

Stockholm, Sweden

N Uexkull

Marselisborg Hospital, Aarhus, Denmark

J Esmann

University Hospital,

Uppsala, Sweden

A Strand

${ }^{\star}$ Members of the group are listed at the end of the

article.

Correspondence to

Dr N J Bodsworth, Sydne

Sexual Health Centre,

Sydney Hospital, Macquarie

Street, Sydney, New South

Wales 2000, Australia.

Accepted for publication

17 December 1996

\title{
Valaciclovir versus aciclovir in patient initiated treatment of recurrent genital herpes: a randomised, double blind clinical trial
}

\author{
N J Bodsworth, R J Crooks, S Borelli, G Vejlsgaard, J Paavonen, A-M Worm, N Uexkull, \\ J Esmann, A Strand, A J Ingamells, A Gibb, and the International Valaciclovir HSV \\ Study Group ${ }^{\star}$
}

Objective: To compare the efficacy and safety of twice daily valaciclovir with five times daily aciclovir in the treatment of an episode of recurrent genital herpes simplex virus (HSV) infection in immunocompetent individuals.

Methods: 739 patients with a history of recurrent genital HSV infection received either oral $\%$ valaciclovir ( $500 \mathrm{mg}$ twice daily) or aciclovir ( $200 \mathrm{mg}$ five times daily) for 5 days for treatment of $\vec{o}$ their next recurrent episode in a controlled, randomised, double blind trial. Patients self initiated therapy at the first signs and/or symptoms of the HSV recurrence, then were assessed in clinic on $\bar{\omega}$ five occasions over 7 days, and twice weekly thereafter until lesions had healed. Safety was eval- $\mathrm{N}$ uated through adverse experience reports and haematology and biochemistry monitoring.

Results: No significant differences were detected between valaciclovir and aciclovir for the pri- iv mary endpoint, the duration of all signs and symptoms which included lesion healing and $\vec{\partial}$ pain/discomfort. The hazard ratio [95\% confidence interval] for valaciclovir $v$ aciclovir was $0.93 \circ$ $[0.79,1.08]$. Lesion healing time was similar in each treatment group (hazard ratio valaciclovir $v ?$ aciclovir $0.96[0.80,1.14])$. The odds ratio of valaciclovir $v$ aciclovir in preventing the develop- $\vec{D}$ ment of vesicular/ulcerative lesions was $1.08[0.82,1 \cdot 42]$. Percentages of patients in whom all HSV cultures were negative were similar in the valaciclovir and aciclovir groups at $59 \%$ and $54 \% \overrightarrow{0}$ respectively; for patients having equal to or more than one positive culture result after treatment $\Theta$ initiation, cessation of virus shedding was similarly rapid for the two treatments (hazard ratio $0.98[0.75,1.27])$. The safety profiles of valaciclovir and aciclovir were comparable with adverse experiences being infrequent and generally mild.

Conclusion: This study has demonstrated that valaciclovir $500 \mathrm{mg}$ twice daily is equivalent in efficacy to aciclovir $200 \mathrm{mg}$ five times daily as episodic treatment of recurrent genital HSV infec- $\bar{\AA}$ tion. Valaciclovir maintains the established efficacy and safety of aciclovir but offers a much $\overrightarrow{\vec{p}}$ more convenient twice daily dosing regimen.

(Genitourin Med 1997;73:110-116)

Keywords: valaciclovir; genital herpes, recurrent; herpes simplex virus

\section{Introduction}

Genital herpes simplex virus (HSV) infection continues to be a major public health problem throughout the world. Epidemiological surveys undertaken in both industrialised and developing countries indicate that the prevalence of HSV infection is rising rapidly, and HSV is now the most common cause of genital ulceration in many countries. ${ }^{1}$ The seroprevalence of HSV-2 is highest in Africa (30-40\%) followed by the USA (13-40\%) and Europe $(7-16 \%){ }^{2}$ Studies show an annual acquisition rate of $2-4 \%$ for HSV-2 depending upon the population studied. ${ }^{2}{ }^{3}$ Over the past decade, aciclovir (Zovirax) has become the therapy of choice for the management of recurrent genital HSV infection. ${ }^{4-6}$ The recommended oral regimen of $200 \mathrm{mg}$ five times daily for five days has been shown to reduce lesion healing time and shorten the course of virus shedding when treatment is initiated within 24 hours of the first signs and/or symptoms of a recurrent HSV episode.$^{7}$ Early treatment may prevent the development of vesicular or ulcerative lesions in some patients if treatment is initiated during the prodrome. ${ }^{8-10}$
Over 15 years of clinical experience has shown aciclovir to be an effective and well 9 tolerated drug for the management of HSV infections. ${ }^{511}$ However, its limited oral bioavailability $(10-20 \%)$ and short plasma $N$ elimination half life mean that frequent dosing $N$ is required. A formulation providing higher $\mathbb{\omega}^{N}$ oral aciclovir bioavailability would therefore improve upon its clinical usefulness. ${ }^{12}$

Valaciclovir (Valtrex, Zelitrex) is the L-valyl ester of aciclovir and once absorbed, is rapidly ? and almost completely converted to aciclovir and the essential amino acid, L-valine after $\mathbb{D}$ oral administration. The absolute bioavailabil- $\mathbb{\Phi}$ ity of aciclovir after oral valaciclovir administration is $54 \%$, a three- to fivefold increase over oral aciclovir itself. ${ }^{1314}$ Two controlledo studies comparing valaciclovir with placebo and with aciclovir and placebo as episodic treatment for recurrent genital HSV infection have shown that both active therapies are effective in speeding resolution of signs and symptoms. ${ }^{15} 16$ The present study was undertaken to investigate whether the efficacy of valaciclovir twice daily could be maintained with the lower unit dose of $500 \mathrm{mg}$. 
Patients and methods

STUDY DESIGN

This was a multicentre, randomised, double blind comparison of twice daily valaciclovir and five times daily aciclovir given for five days for the treatment of a single episode of recurrent genital herpes infection. For the purpose of this study, genital herpes was defined as a history of recurrent HSV infection involving genital, perianal, or closely related sites.

\section{PATIENTS}

Male and female patients of at least 18 years of age were eligible to enter the study if they had experienced four or more recurrences of genital HSV reactivation in the previous 12 months. Those who had been previously receiving suppressive aciclovir had to have experienced a recurrence within three months of stopping therapy and within the three months before enrolment into the study in order to be eligible. Patients were not eligible if they had significant hepatic or renal impairment, were pregnant or nursing mothers, had malabsorption syndrome, were immunocompromised, known to be HIV seropositive, or were receiving probenecid, other systemic antiviral medications, or immunomodulatory treatment.

Ethics committee approval was obtained at each study site before recruitment commenced and all patients gave full written informed consent.

\section{STUDY PROCEDURES}

Eligible patients were screened then randomised in equal numbers to one of two treatment arms - oral valaciclovir $500 \mathrm{mg}$ twice daily or oral aciclovir $200 \mathrm{mg}$ five times daily for five days. A blood sample was taken at the screening visit for baseline haematology and clinical chemistry, and to determine HSV-2 serological status if there was no record of a positive HSV culture during the study or a documented history of culture proved genital HSV infection in the patient's usual medical record. Serological testing was by western blot at a single reference laboratory. Pregnancy tests were performed on all females of childbearing potential at screening and again on the first day of treatment (day 1).

Study medication was dispensed at screening. Patients randomised to valaciclovir also received placebo (dummy) aciclovir tablets; those randomised to aciclovir also received placebo valaciclovir tablets.

Patients self initiated treatment at the first signs or symptoms of their next recurrence. Signs and symptoms included mucocutaneous lesions and/or pain or discomfort. They attended the clinic for evaluation within 24 hours of starting medication. Clinical evaluation and staging of external lesions (macule/ papule, vesicle/ulcer, crust, or healed) was performed on days $1,2,3,5$, and 7 . Patients attended the clinic twice weekly thereafter if lesions had not healed by day 7. Diaries were used throughout the treatment and follow up period for patients to record the date and time of prodrome/first signs of genital lesions, compliance with the dosing schedule, the patient's own assessment of lesion healing, and the pain/discomfort level (no pain, mild, moderate, or severe pain). Swabs for HSV culture were taken at each clinic visit until all lesions had healed. Virus isolation was performed at a single reference laboratory in each city according to local protocol. Safety was assessed by adverse experience reporting at each visit and from haematology (haemoglobin, white blood cell, and platelet counts) and clinical chemistry (creatinine, alkaline phosphatase, and AST or ALT) evaluations on days 1 and 5 . Blood was drawn for determination of steady state plasma aciclovir concentrations by radioimmunoassay on days 2 and $5 .{ }^{17}$ Compliance was assessed by pill count of returned blister packs. All patients who did not start treatment were asked to return unused study medication.

\section{EFFICACY ENDPOINTS}

The primary efficacy endpoint on which the sample size calculation was based was length of episode, which was defined as the number of days from treatment initiation to complete resolution of all signs and symptoms. In the majority of patients, genital HSV lesions were expected to progress through the usual stages of vesiculation or ulceration, and scabbing before healing. ${ }^{15}$ Lesion healing time was therefore also considered a primary efficacy endpoint and was defined as the number of days between initiation of treatment and complete re-epithelialisation of all mucocutaneous lesions. Secondary endpoints were whether or not the HSV episode aborted (defined by the presence of symptoms such as pain/discomfort but failure of lesions to develop beyond the macule/papule stage), duration and severity of pain/discomfort, percentages of patients having no positive HSV culture after treatment initiation, and duration of virus shedding in those patients having at least one positive HSV culture.

\section{SAMPLE SIZE CALCULATION}

The minimum sample size of 250 treated patients per arm provided sufficient power to detect differences between treatments in the duration of all signs and symptoms, assuming proportional hazards functions. The sample size provided $80 \%$ power to detect hazard ratios between 0.76 and 1.31 at the $5 \%$ level of significance, assuming that $45 \%$ of the aciclovir group would have signs or symptoms at day five. This is equivalent to detecting an arithmetic reduction of $10 \%$ (45\% to $35 \%)$ in the proportion of patients still having signs or symptoms at day 5 . Smaller treatment differences were considered clinically unimportant; therefore, if the resulting $95 \%$ confidence intervals for the hazard ratio [95\% CI] fell within these clinically unimportant ranges, the power of the study was such that equivalence could be concluded.

\section{STATISTICAL ANALYSIS}

The data listing and statistical analyses were performed using Statistical Analysis Systems software version 6.07 (SAS Institute Inc, 
Cary, NC, USA). The intent to treat analysis group was defined as all patients randomised who returned to the clinic for assessment of an HSV recurrence. This group was used for the analysis of the primary efficacy endpoint of episodic length and relevant secondary endpoints, as well as for exploratory analyses and the evaluation of safety. Patients in whom vesicular/ulcerative lesions were prevented were excluded from the analysis of lesion healing time. Patients for whom there was no positive HSV culture result during treatment or follow up were excluded from the analysis of duration of virus shedding.

The distributions of time to event endpoints (length of episode, lesion healing time, duration of pain/discomfort, and duration of viral shedding) were estimated using the KaplanMeier product limit method. With the exception of duration of viral shedding, all other times to event were calculated in hours, from signs or symptom onset as recorded in patient diaries. Cox's proportional hazards models were used to estimate treatment differences. Formal hypothesis testing was performed for length of episode. The Cochran-MantelHaenszel test was used to investigate treatment differences in the percentages of patients with pain/discomfort on days three and seven and in those in whom vesicular/ulcerative lesions were prevented. Odds ratios were also derived for percentages of patients in whom lesions failed to progress to vesicles/ulcers. Since previous studies have identified sex as an important factor influencing efficacy, ${ }^{18} 19$ it was included as a covariate in the Cox's proportional hazard models or logistic regression models as appropriate. Hodges-Lehmann estimates $[95 \% \mathrm{CI}]$ were used to assess any treatment differences or changes over time in haematology and biochemistry variables. All adverse experience data were tabulated and compared across the two treatment groups.

Additional exploratory analyses were conducted on episode length, lesion healing time, and percentages of patients in whom vesicular/ulcerative lesions were prevented. Age (years) and time from prodrome/first sign to initiation of therapy (hours) were fitted as continuous variables; prior use of suppressive aciclovir therapy (used $v$ not used), and number of recurrences in the previous year $(\leqslant 8 v$

Table 1 Demographic characteristics and HSV disease history

\begin{tabular}{lll}
\hline & $\begin{array}{l}\text { Valaciclovir } \\
(n=378)\end{array}$ & $\begin{array}{l}\text { Aciclovir } \\
(n=361)\end{array}$ \\
\hline $\begin{array}{l}\text { Median age (years) (range) } \\
\text { Sex (\%) male/female }\end{array}$ & $32(18-77)$ & $33(20-74)$ \\
Median time from first genital HSV & $50 / 50$ & $49 / 51$ \\
$\quad$ episode (years) & $3 \cdot 66$ & $4 \cdot 15$ \\
No of recurrences in previous year (\%) & 3 & 3 \\
$\quad$ 1-3 & 60 & 68 \\
$4-8$ & 37 & 29 \\
$\geqslant 9$ & $17 \%$ & $14 \%$ \\
Use of suppressive aciclovir in previous year & $0 \cdot 34$ & $0 \cdot 34$ \\
Median time on suppressive therapy (years) & & $25 \cdot 5 \%$ \\
HSV culture before or at screening & $29 \cdot 9 \%$ & $1 \cdot 7 \%$ \\
HSV-2 & $0 \cdot 8 \%$ & $12 \cdot 7 \%$ \\
HSV-1 & $11 \cdot 6 \%$ & $60 \cdot 1 \%$ \\
Not typed & $57 \cdot 7 \%$ & \\
HSV-2 infection confirmed by serology & & \\
\hline
\end{tabular}

^Performed on patients without documented positive HSV culture at genital site. $\geqslant 9)$ were also incorporated as covariates into the models in order to identify their impact on the clinical endpoints.

\section{PLASMA ACICLOVIR LEVEL MONITORING AND} PHARMACOKINETIC ANALYSIS

Plasma aciclovir concentrations for subjects in $\mathbb{\Phi}$ each treatment group were plotted as a function of time after the last dose. Mean concen- $\subseteq$ tration $v$ time profiles for valaciclovir and aciclovir recipients were estimated using nonlinear mixed effect modelling (NONMEM version 4$)^{20}$

\section{Results}

PATIENT CHARACTERISTICS

In all, 999 patients were randomised to treatment at 48 study sites in Europe and $\vec{\circ}$ Australia. The intent to treat group consisted of 739 patients who returned for clinic assess- $\vec{\omega}$ ment. Overall, $59 \%$ of treated patients did note have a documented positive culture for $\mathrm{HSV}_{\omega}$ at a genital site and required serological confirmation (table 1). In total, 378 patients were treated with valaciclovir and 361 received aciclovir. Those patients who did not return to the clinic were unlikely to have had a recur- $\vec{D}$ rence and therefore would not have com-음 menced study drug treatment.

The demographic characteristics and HSV disease history of treated subjects at screening. are shown in table 1 and were similar in the valaciclovir and aciclovir groups. The sex split was equal in both groups; ages ranged from $18 \stackrel{\circ}{\circ}$ to 77 years (median 32 years). HSV disease history was similar in the valaciclovir and aciclovir groups although slightly more patients in the valaciclovir group had experienced $\geqslant 9$ recurrences in the previous 12 months compared with the aciclovir group ( $37 \% v 29 \%)$. The median time from the initial HSV episode $\bar{\sigma}$ was approximately four years. Overall,,$\frac{3}{\text {. }}$ $14 \%-17 \%$ had received suppressive acicloviro therapy in the previous year for a median time of four months. A positive HSV culture was 9 recorded for $41 \%$ of patients before enrolment. The slight imbalance noted in previous HSV recurrence frequency necessitated routine inclusion of this factor as a covariate in $N$ the efficacy analyses. There were insufficient numbers of patients with proved HSV-1 infec- tion $(1 \%-2 \%)$ for separate analysis.

ANALYSIS OF EFFICACY

Length of episode

No significant difference between valaciclovir and aciclovir was detected in length of episode, as evidenced by the hazard ratio $[95 \% \mathrm{CI}]$ of $0.93[0.79,1.08] . p=0.34$ (table 2). The median episode duration for the valaciclovir and aciclovir groups was 4.7 and $\lesssim$ $4 \cdot 6$ days respectively (fig 1 ).

\section{Lesion healing}

In those patients with lesions that progressed to vesicles/ulcers, complete re-epithelialisation occurred as rapidly with valaciclovir as aciclovir. The hazard ratio $[95 \% \mathrm{CI}]$ for valaciclovir $v$ aciclovir was $0.96[0.80,1.14]$ (table 
Table 2 Valaciclovir versus aciclovir for recurrent genital herpes: time to event endpoints

\begin{tabular}{|c|c|c|}
\hline & \multicolumn{2}{|l|}{ Valaciclovir v aciclovir } \\
\hline & $\begin{array}{l}\text { Hazard ratio } \\
(95 \% \text { CI })\end{array}$ & $\begin{array}{l}\text { Median time to } \\
\text { event (days) }\end{array}$ \\
\hline $\begin{array}{l}\text { Length of episode }{ }^{\star} \\
\text { Lesion healingt }\end{array}$ & $\begin{array}{l}0.93(0.79,1.08) p=0.34 \\
0.96(0.80,1.14)\end{array}$ & $\begin{array}{l}4 \cdot 7 v 4 \cdot 6 \\
4 \cdot 4 v 4 \cdot 5\end{array}$ \\
\hline $\begin{array}{l}\text { Duration of pain/discomfort } \$ \text { : } \\
\text { overall } \\
\text { males } \\
\text { females }\end{array}$ & $\begin{array}{l}0.91(0.78,1.06) \\
0.89(0.71,1.12) \\
0.97(0.77,1.22)\end{array}$ & 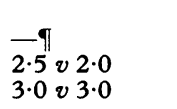 \\
\hline $\begin{array}{l}\text { Duration of virus shedding§: } \\
\text { overall } \\
\text { males } \\
\text { females }\end{array}$ & $\begin{array}{l}0.98(0.75,1.27) \\
0.93(0.65,1.33) \\
0.95(0.61,1.47)\end{array}$ & 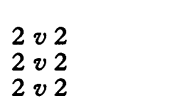 \\
\hline
\end{tabular}

*Time from treatment initiation to resolution of all signs and symptoms $(n=710)$. tDefined as loss of crusts and re-epithelialisation, includes only patients who developed vesicular/ulcerative lesions $(n=559)$

$\ddagger$ All patients $(n=712)$.

Patients with $\geqslant 1$ positive HSV culture $(n=295)$

TProportional hazards assumption not satisfied for sex, therefore separate analyses of males and females was necessary.

Figure 1 Kaplan-Meier plot of percentages of patients with signs or herpes recurrence following treatment with valaciclovir or aciclovir. symptoms of a genital

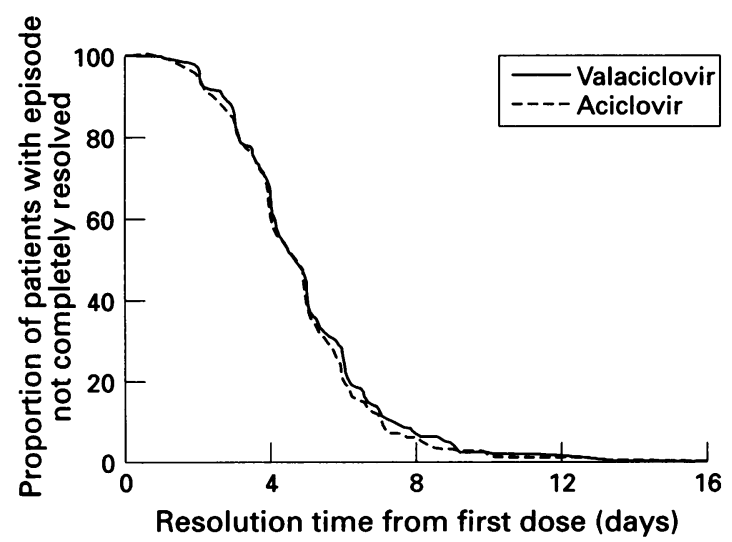

Figure 2 Kaplan-Meier plot of percentages of patients with cutaneous lesions of a genital herpes recurrence not yet healed following treatment with valaciclovir or aciclovir.

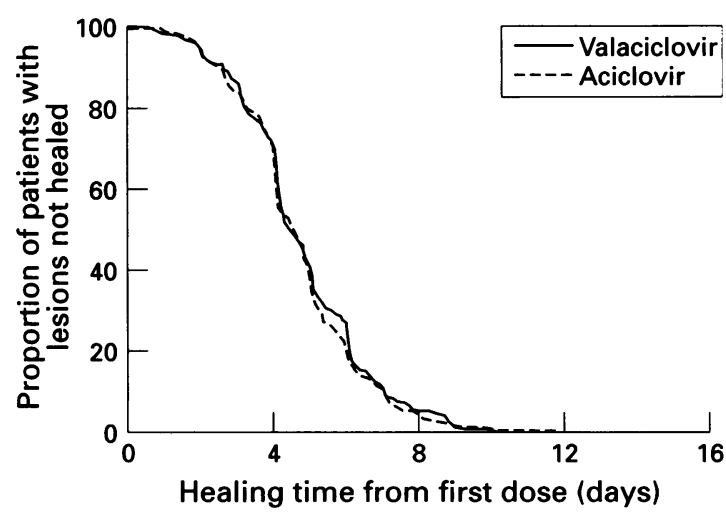

Table 3 Valaciclovir versus aciclovir for recurrent genital herpes: prevention of lesion development, pain, and virus shedding proportions

\begin{tabular}{|c|c|c|c|}
\hline & Valaciclovir & Aciclovir & $\begin{array}{l}\text { Valaciclovir v } \\
\text { aciclovir } \\
\text { Relative risk } \\
(95 \% \text { CI) }\end{array}$ \\
\hline \multicolumn{4}{|l|}{ Percentage in whom vesicular/ulcerative } \\
\hline overall & 22 & 21 & $1.08(0.82,1.42)$ \\
\hline males & 20 & 18 & $1.26(0.82,1.93)$ \\
\hline females & 24 & 24 & $0.96(0.67,1.37)$ \\
\hline \multicolumn{4}{|l|}{ Percentage with pain on day $3^{\star}$} \\
\hline none & 40 & 42 & $0.30 \dagger$ \\
\hline mild & 43 & 42 & \\
\hline moderate/severe & 11 & 11 & \\
\hline \multicolumn{4}{|l|}{ Percentage with pain on day $7 \dagger$} \\
\hline none & 50 & 52 & $0.87 \dagger$ \\
\hline mild & 4 & 2 & \\
\hline moderate/severe & 1 & 1 & \\
\hline Percentage with no positive HSV culture & 59 & 54 & ND \\
\hline
\end{tabular}

$\star$ Pain scores missing for some patients.

†Cochran-Mantel-Haenszel statistic.

$\mathrm{ND}=$ not determined
2). Median healing times were $4 \cdot 4$ and 4.5 days for the valaciclovir and aciclovir groups respectively (fig 2 ).

Prevention of vesicular/ulcerative lesions

The percentages of patients in the valaciclovir and aciclovir groups in whom vesicular/ulcerative lesions were prevented was similar, at $22 \%$ and $21 \%$ respectively, and did not differ with sex (table 3). The odds ratio [95\% CI] for the chance of vesicular lesions being prevented with valaciclovir treatment compared with aciclovir was $1.08[0.82,1.42]$.

\section{Pain/discomfort}

No differences between valaciclovir and aciclovir were evident in the percentages of patients experiencing no/mild pain or moderate/severe pain on day 3 or day 7 (table 3 ). Resolution of pain/discomfort was achieved as rapidly with valaciclovir as aciclovir in all patients and in males and females when analysed separately. Hazard ratios [95\% CI] indicated no treatment differences (table 2).

\section{Virus shedding}

In patients with at least one swab for HSV culture, results were all negative in similar proportions in each treatment group, at $59 \%$ for valaciclovir and $54 \%$ for aciclovir (table 3 ). For those in whom at least one culture was positive during treatment or follow up, termination of virus shedding was as rapid in each group, as evidenced by the hazard ratio [ $95 \%$ CI] for valaciclovir $v$ aciclovir of $0.97[0.75$, 1.26], with no differences being apparent between the sexes (table 2).

\section{Exploratory analyses}

Results of the exploratory analyses suggest that episode resolution was $20 \%$ faster in those patients who had previously received suppressive aciclovir therapy in the year before enrolment compared with those who had not (hazard ratio $[95 \% \mathrm{CI}] 0.80[0.63,1.01] \mathrm{p}=$ $0 \cdot 06)$. Earlier treatment initiation accelerated episode resolution (hazard ratio for the difference $[95 \% \mathrm{CI}] 0.99[0.98,1.00] \mathrm{p}=0.01$ ) and lesion healing (hazard ratio for the difference $[95 \% \mathrm{CI}] 0.99[0.98,1.00] \mathrm{p}=0.03)$. Hence, in patients starting treatment six hours after first signs/symptoms, episode resolution and lesion healing were $15 \%$ and $13 \%$ faster, respectively, than in those starting treatment 24 hours after first signs/symptoms.

ANALYSIS OF SAFETY

Adverse experiences reported during treat-

Table 4 Adverse experiences reported by $\geqslant 2 \%$ of patients

\begin{tabular}{lll}
\hline Adverse experience & $\begin{array}{l}\text { Valaciclovir } \\
n=378 \\
(\%)\end{array}$ & $\begin{array}{l}\text { Aciclovir } \\
n=361 \\
(\%)\end{array}$ \\
\hline Headache & 14 & 9 \\
Nausea & 8 & 8 \\
Diarrhoea & 2 & 5 \\
Abdominal pain & 3 & 3 \\
Asthenia & 3 & 3 \\
Dizziness & 2 & 2 \\
Insomnia & 2 & 1 \\
\hline
\end{tabular}


ment and follow up, irrespective of association, which occurred in $\geqslant 2 \%$ of subjects, are listed in table 4 . The most frequent were headache and nausea; no major differences between treatments were evident. Headache was considered possibly attributable to valaciclovir or aciclovir in $10 \%$ and $7 \%$ of patients, with nausea thought possibly attributable in $8 \%$ and $7 \%$, respectively. Three adverse experiences in three patients were considered serious, but only one, a patient receiving aciclovir who suffered abdominal pain, was thought possibly attributable to the drug. Treatment was discontinued prematurely in five patients (valaciclovir three, aciclovir two) as a result of adverse experiences.

There were no clinically important changes from screening in any haematology or clinical chemistry variable with valaciclovir or aciclovir treatment or differences between groups during treatment or between values from samples taken on day one and day five. Mean serum creatinine values (SD) on day one were 87.9 (14.7) and $87 \cdot 2(13 \cdot 2) \mu \mathrm{M}$ for the valaciclovir and aciclovir groups, respectively. On day five, respective mean values were $87.9(14 \cdot 1)$ and $87.5(13 \cdot 8) \mu \mathrm{M}$, indicating no meaningful differences between the groups or with treatment.

PLASMA ACICLOVIR LEVEL MONITORING Estimated mean peak aciclovir concentrations for patients randomised to valaciclovir and aciclovir were $12.3 \mu \mathrm{M}(2.8 \mu \mathrm{g} / \mathrm{ml})$ and $2.9 \mu \mathrm{M}$ $(0.65 \mu \mathrm{g} / \mathrm{ml})$ respectively. Corresponding estimates of the areas under the aciclovir plasma concentration $v$ time curves over 24 hours were $99.5 \mu \mathrm{M} / \mathrm{h}(22.4 \mathrm{~h} / \mu \mathrm{g} / \mathrm{ml})$ and $49.3 \mu \mathrm{M} / \mathrm{h}$ $(11 \cdot 1 \mathrm{~h} / \mu \mathrm{g} / \mathrm{ml})$. Thus, the $500 \mathrm{mg}$ valaciclovir regimen increased the systemic exposure to aciclovir by twofold over the standard oral aciclovir regimen. Since a $500 \mathrm{mg}$ dose of valaciclovir contains approximately $347 \mathrm{mg}$ aciclovir, the bioavailability of aciclovir from valaciclovir $500 \mathrm{mg}$ twice daily was 2.9 times greater than that from aciclovir $200 \mathrm{mg}$ five times daily in this study.

\section{Discussion}

This study has found that valaciclovir at a dose of $500 \mathrm{mg}$ twice daily for five days and aciclovir at the standard dose of $200 \mathrm{mg}$ five times daily also for five days are equally effective in patient initiated treatment of recurrent genital herpes. Moreover, this clinical equivalence is achieved without apparent compromise to the excellent safety profile established for aciclovir. This was one of two large trials conducted to examine a $500 \mathrm{mg}$ twice daily regimen of valaciclovir, after an earlier placebo controlled study of similar design demonstrated that $1000 \mathrm{mg}$ was as effective as the standard aciclovir regimen in shortening all signs and symptoms of a recurrence. ${ }^{16}$ The companion study has shown $500 \mathrm{mg}$ and 1000 $\mathrm{mg}$ unit doses of valaciclovir taken twice daily to be equivalent for the same efficacy endpoints and both to be superior to placebo. ${ }^{15}$ From this and the results of the present study, it may be concluded that valaciclovir $500 \mathrm{mg}$ twice daily achieves the same clinical efficacy in treating genital herpes recurrences as the standard oral aciclovir regimen.

The five times a day dosing regimen for aciclovir for recurrent genital herpes has been supported by several clinical studies 89162122 ( and is currently recommended by the US Centers for Disease Control and Prevention. ${ }^{6}$ An effective twice daily treatment regimen for recurrent genital herpes may confer benefits in addition to the greater convenience and improved compliance usually attributed to $\overrightarrow{\vec{\rho}}$ simplified dosing schedules. There is a degree of general community awareness of the indications for aciclovir (Zovirax). Consequently, $\frac{\bar{\omega}}{\vec{D}}$ many patients with anxiety about the potential $\triangle$ social consequences of their diagnosis, includ- is ing fear of detection, will appreciate a regimen $\vec{\circ}$ that allows all treatment to be taken (and stored) in the privacy of the home.

Simulated plasma aciclovir concentration $v$ on time profiles indicated that the $500 \mathrm{mg}$ twice daily valaciclovir regimen would increase sys- i temic aciclovir exposure by 1.5 to 2 -fold compared with that following oral aciclovir. ${ }^{13} \vec{\circ}$ Plasma aciclovir level monitoring in this study 을 in young adults with genital herpes, a population of similar age to that in the formal phase 1 음 study, has confirmed that at steady state, $500 \mathrm{mg}$ twice daily valaciclovir does result in the predicted doubling of aciclovir exposure.

The results of this study have demonstrated both the clinical and statistical equivalence in efficacy of valaciclovir and the standard oral aciclovir regimen on the primary endpoint. We $\frac{0}{\mathbb{2}}$ believe that the study was adequately protected against type 2 error; a $10 \%$ difference in the number of patients with signs or symptoms at day 5 could have been detected with $80 \%$ ? power. Although not statistically powered for 0 hypothesis testing on all the other efficacy endpoints, analyses consistently indicated in each 3 case no evidence for any meaningful differences between treatment groups. Of particular clinical importance are the results showing 의 that vesicular lesions were prevented in similar percentages of patients and that in $59 \%$ and $54 \%$ of valaciclovir and aciclovir recipients, $\widetilde{N}$ respectively, no HSV culture was positive once $N$ treatment had begun.

The primary efficacy endpoint, length of episode, encompasses all clinical signs and 0 symptoms, specifically the more traditional measure of cutaneous lesion healing plus resolution of pain, itching, or other discomfort. The intent to treat population, on which the primary time to event analysis of length of $\frac{\pi}{\mathbb{D}}$ episode was based, thus comprises two sub- $\frac{\Omega}{0}$ populations defined according to clinical outcome-those in whom clinically evident skin lesions progress through all stages of healing and those whose initial symptoms indicated a pending recurrence but which subsequently fails to develop beyond the papule stage (aborted episode). Lesions that fail to progress have been recognised in several earlier clinical trials of aciclovir and represent the most desirable clinical outcome. ${ }^{8-10}$

Patient initiated treatment is designed to 
minimise the time from onset of symptoms to commencement of therapy. Studies comparing patient and physician initiated treatment for acute episodes of genital herpes have supported the patient initiated approach for oral aciclovir. $^{818}$ Exploratory analyses in the present study lend further support for this strategy, having shown that both episode duration and lesion healing are briefer with earlier treatment. However, the time from symptom onset to starting treatment did not influence the number of patients in whom vesicular lesions were prevented. One possible explanation may be the protocol requirement for patients to initiate treatment within 24 hours of any symptom of a recurrence, including skin lesions alone; this would not necessarily ensure prodromal treatment and would not optimise the chances of even earlier termination of $\mathrm{HSV}$ replication and prevention of skin damage. Future studies to address this desirable clinical outcome should include patients with prodrome only.

We found that in patients who had used suppressive aciclovir therapy in the 12 months before enrolment, resolution of the episode was on average $20 \%$ faster than in patients who had not. An explanation for this is not readily apparent as suppressive therapy is considered to affect the course of HSV disease only during the period of treatment and the subsequent recurrence rate is not affected even by prolonged therapy. ${ }^{192324}$ The availability of aciclovir for long term suppression of genital herpes is such that studies of its long term benefits have only been possible for a maximum period of 10 years. ${ }^{22} 26$ For an infection such as genital HSV that persists throughout life, and as evidenced by the current study in which the oldest patients were 77 years of age, 10 years is probably not an adequate period on which to draw conclusions regarding the natural history.

The safety profile of aciclovir has been carefully established over more than 12 years of clinical use. ${ }^{511}$ In the current study, there were no clinically significant differences in the nature, frequency, or severity of adverse events between treatment groups, and no haematology or clinical chemistry variable was significantly affected during treatment. Previous placebo controlled trials of aciclovir or valaciclovir in the acute treatment of recurrent genital herpes in immunocompetent adults have shown that adverse events occurred in similar percentages of patients receiving the active treatments and placebo, suggesting that such events are typical of the disease rather than a result of therapy with aciclovir or valaciclovir. ${ }^{7891516}$ Although the daily area under the plasma aciclovir concentration $v$ time curve after valaciclovir was twice that following aciclovir, and peak plasma concentrations were approximately four times greater after valaciclovir, no meaningful changes in serum creatinine values were detected during treatment with valaciclovir or aciclovir.

The present study has demonstrated that twice daily valaciclovir is as effective as the standard five time daily aciclovir regimen in rapidly resolving the signs and symptoms of an episode of recurrent genital herpes. Valaciclovir confers all the clinical benefits of aciclovir but with the convenience and security of a twice daily dosing regimen and without apparent compromise to aciclovir's excellent safety profile.

The authors and the International Valaciclovir HSV Study Group gratefully acknowledge the support of GlaxoWellcome for assistance with the conduct of this study. We are particularly indebted to $M$ Jones for statistical analysis, $S$ Weller for pharmacokinetic analysis, and A R Bell for his contribution to the preparation of the manuscript.

\section{The International Valaciclovir HSV Study Group}

$S$ Barton, Chelsea and Westminster Hospital, London, UK; C Beylot, Hopital Due Haute-leveque, Pessac, France; J Bingham, St Thomas' Hospital, London, UK; N J Bodsworth, Sydney Hospital and Taylor Square Private Clinic, Darlinghurst, Sydney, Australia; G Bojs, Central Hospital, Kristianstad, Sweden; S Borelli, Dermatologische Klinik und Poliklinik der TU, Munich, Germany; D Cheetham, Ealing General Hospital, Southall, UK; E Curless, Bolton General Hospital, Bolton, UK; B Czarnetzki, Universitatsklinikum, Rudolf Virchow, Berlin, Germany; S Davies, Port Kembla Hospital, Warrawong, Australia; A Eichmann, Dermatology and Venereology Disease Clinic, Zurich, Switzerland; J Esmann, Marselisborg Hospital, Aahus, Denmark; B Goh, The London Hospital, London, UK; D Goldmeir, St Mary's Hospital, London, UK; G Gross, Universitats Hautklinik und Poliklinik der TU, Hamburg, Germany; U F Haustein, Hautkinik der Universitat, Leipzig, Germany; G Kinghorn, Royal Hallamshire Hospital, Sheffield, UK; J Lauharanta, Helsinki University, Central Hospital, Finland; C Law, St George Hospital, Kogarah, Australia; G Luzzi, Wycombe General Hospital, High Wycombe, UK; A McMillan, Edinburgh Royal Infirmary, Edinburgh, UK; J Meaden, Norfolk and Norwich Hospital, Norfolk, UK; U Montemagno, Chirguia Universita, Napoli, Italy; P Morel, Hopital Saint Louis, Paris, France; M Negosanti, Universita degli Studi, Bologna, Italy; E Nielsen, Esbjerg Hospital, Esbjerg, Denmark; A Nilsen, Haukeland Hospital, Bergen, Norway; E Ong, Newcastle General Hospital, Newcastle, UK; J P Ortonne, Hopital Pasteur, Nice, France; J Paavonen, Helsinki University Central Hospital, Finland; R Patel, Royal South Hants Hospital, Southhampton, UK; J Patten, Brisbane Special Clinic, Australia; D Petzold, Heidelberg University, Germany; T Rufli, Kantonsspital, Basel, Switzerland; S Saari, Laakariasema, Turku, Finland; $M$ Shahmanesh, Birmingham General Hospital, UK; A Simpanen, Laboratorio Simpanen, Kupio, Finland; J Soltz-Szots, Hospital Rudolfstiftung, Vienna, Austria; J P Stahl, CHU de Grenoble, Grenoble, France; E Stolz, University Hospital, Rotterdam, Netherlands; A Strand, Akademiska Hospital, Uppsala, Sweden; I Thelin, Lund Hospital, Lund, Sweden; G Vejlsgaard, Rigshopitalet, Copenhagen, Denmark; N Von Uexkull, Citymottagningen, Stockholm, Sweden; A Wikstrom, Karolinska Hospital, Stockholm, Sweden; P Woolley, Withington Hospital, Manchester, UK; A-M Worm, Bispebjerg Hospital, Copenhagen, Denmark.

1 Corey L. Genital herpes. In: Holmes KK, Mardh PA, Sparling PF, Weisner PJ, eds. Sexually transmitted diseases. Part 5 Sexually transmitted agents. New York: McGraw-Hill, 1990.

2 Nahmias AJ, Lee FK, Beckman-Nahmias $S$ Seroepidemiological and sociological patterns of herpes simplex virus infection in the world. Scand $\mathcal{F}$ Infect Dis simplex virus infection
Suppl 1990;69:19-36.

3 Johnson RE, Nahamias AJ, Magder LS, Lee FK, Brooks CA, Snowden CB. A seroepidemiological survey of the prevalence of herpes simplex virus type-2 infection in the United States. N Engl f Med 1989;321:7-12.

4 O'Brien JJ, Campoli-Richards DM. Acyclovir-an updated review of its anti-viral activity, pharmacokinetic properties and therapeutic efficacy. Drugs 1989;37:233-309. 
5 Whitley RJ, Gnann JW. Acyclovir: A decade later. $N$ Engl $\mathcal{F}$ Med 1992;327:782-9.

6 Centers for Disease Control and Prevention. Sexually transmitted diseases treatment guidelines. MMWR 1993; 42(RR-14):23.

7 Goldberg LH. Oral acyclovir for episodic treatment of recurrent genital herpes: efficacy and safety. $\mathcal{F} \mathrm{Am} \mathrm{Acad}$ Dermatol 1986;15:256-64.

8 Reichman RC, Badger GL, Mertz GL, Corey L, Richman $\mathrm{DD}$, Connor $\mathrm{JD}$, et al. Treatment of recurrent genital herpes simplex infections with oral acyclovir. I $A M A$ 1984;251:2103-7.

9 Ruhnek-Forsbeck M, Sandstrom E, Andersson B, Eriksson G, Hersle K, Lovhagen GB, et al. Treatment of recurrent $\mathrm{G}$, Hersle $\mathrm{K}$, Lovhagen $\mathrm{GB}$, et al. Treatment of recurrent genital herpes simplex infections with
$\Im$ Antimicrob Chemother 1985;16:621-8.

10 Whatley JD, Thin RN. Episodic acyclovir therapy to abort recurrent attacks of genital herpes simplex infection. f Antimicrob Chemother 1991;27:677-81.

11 Tilson HH, Engle CR, Andrews EB. Safety of acyclovir: a summary of the first 10 years' experience. $₹$ Med Virol 1993;1:67-73.

12 Beachamp LM, Krenitsky TA. Acyclovir prodrugs: the road to valaciclovir. Drugs of the Future 1993;18: 619-28.

13 Weller S, Blum MR, Doucette M, Burnette T, Cederberg $\mathrm{DM}$, de Miranda $\mathrm{P}$, et al. Pharmacokinetics of the acyclovir prodrug valaciclovir after single- and multiple-dose administration to normal volunteers. Clin Pharmacol Exp Ther 1993;54:595-605.

14 Soul-Lawton J, Seaber E, On N, Wootton R, Rolan P, Posner J, et al. Absolute bioavailability and metabolic disposition of valaciclovir, the L-valyl ester of acyclovir, following oral administration to humans. Antimicrob Agents Chemother 1995;39:2759-64.

15 Spruance SL, Tyring SK, Degregorio B, Miller C, Beutner K. A large scale placebo-controlled trial of peroral valaciclovir for episodic treatment of recurrent herpes genitalis. clovir for episodic treatment of recu

16 The International Valaciclovir HSV Study Group, Smiley ML. Valaciclovir and acyclovir for the treatment of recurrent genital herpes simplex virus infections. 33rd Interscience Conference on Antimicrobial Agents and Chemotherapy, October 1993, USA
17 Quinn RP, Tadepalli S, Orban BS, Gerald L. Immunoassay procedures, including RIA for detection of antiviral compounds: Zovirax and Retrovir as examples, pp 185-94. In: Reid E, Wilson I, eds. Methodological surveys in biocehmistry and analysis, vol 20: analysis for surveys in biocehmistry and analysis, vol 20: analysis for Cambridge: Royal Society of Chemistry, 1990.

18 Goldberg LH, Kaufman R, Conant ME, Sperber J, Allen ML, Illeman $M$, et al. Episodic twice-daily treatment for recurrent genital herpes. $A m \mathcal{F}$ Med 1988;85 (Suppl 2a): 10-3.

19 Mattison HR, Reichman RC, Benedetti J, Bolgiano D, Davis LG, Bailey-Farchione A, et al. Double-blind, placebo-controlled trial comparing long-term suppressive with short-term oral acyclovir therapy for management of recurrent genital herpes. Am $\mathcal{F}$ Med 1988;85 (Suppl 2a): 20-4.

20 Beal SL, Sheiner LB. NONMEM User's Guide. San Francisco: University of California at San Francisco, 1989.

21 Nilson AE, Aasen T, Halsos AM, Kinge BR, Tjotta EA, Wikstrom $\mathrm{K}$, et al. Efficacy of oral acyclovir in the treatment of initial and recurrent genital herpes. Lancet 1982;ii:571-3.

22 Salo OP, Lassus A, Hovi R, Fiddian AP. Double blind placebo-controlled trial of oral acyclovir in recurrent genital herpes. Eur $\mathcal{F}$ Sex Transm Dis 1983;1:95-8.

23 Corey L, Mindel A, Fife KH, Sutherland S, Benedetti JW, Adler MW. Risk of recurrences after treatment of first episode genital herpes with intravenous acyclovir. Sex Transm Dis 1985;12:215-8.

24 Mindel A, Weller IVD, Faherty A, Sutherland S, Fiddian AP, Adler MW. Acyclovir in first attacks of genital herpes and prevention of recurrences. Genitourin Med 1986;62: 28-32.

25 Goldberg LH, Kaufman R, Kurtz TO, Conant MA, Eron T LJ, Batenhorst RL, et al. Long-term suppression of recurrent genital herpes with acyclovir. Arch Dermatol 1993; $\overrightarrow{0}$ rent genital hi

26 Baker DA, Safrin S, Deeter RG, Walker A, Barton G, and the Acyclovir Study Group. Nine year effectiveness of continuous suppressive therapy with acyclovir (ACV) in $I$ patients with recurrent genital herpes (RGH). F Eur Acad Dermatol Venereol 1995;5 (Suppl 1):S169.

\section{Historical vignette}

\section{Gram's stain}

Hans Christian Joachim Gram was born in Copenhagen in 1853, the son of a professor of law. $\mathrm{He}$ qualified in medicine in 1878, and after holding junior posts he joined a course in microbiology. His tutor formed a high opinion of him, and asked Carl Friedländer if he could study in his laboratory in Berlin. He arrived there in 1883, and almost at once embarked on an investigation into the problem of identifying bacteria in stained tissue sections. The techniques in use at this time made this difficult because cell nuclei, fibrin, and bacteria stained with equal intensity. Gram found that if he applied a solution of iodine and potassium iodide to sections already stained with gentian violet, then immersed them in absolute alcohol, he could decolorise the tissue cells without changing the deep blue colour of many bacteria. Subsequently, he found it useful to counterstain the tissue with Bismarck or aniline brown.

Three weeks after his arrival in Berlin, Gram wrote to his tutor in Copenhagen: "I have had the good luck to find what seems to be a very good method of staining cocci while the tissue and cell nuclei remain unstained. Dr F is delighted with the method." Friedländer soon mentioned Gram's method in a paper on the micrococci of pneumonia, then Gram himself published a detailed account of it. By this time, he had found that it could be applied to smears as well as tissues, so that it was possible to distinguish bacteria which retained their blue colour after the iodine/alcohol treatment ("Gram positive") from those which were decolorised ("Gram negative"). He ended his paper with these words: "Hopefully, the method will prove useful in the hands of other investigators." Having said this, he did no fur- ther work on the technique, leaving its refinement to others. Having been in Berlin for only five months, he $\mathrm{o}$ returned to Copenhagen, where in due course he became professor of pharmacology and senior physi- $\exists$ cian at Frederik Hospital. He died in 1938 at the age of 85 years.

Neisser first identified the gonococcus in 1879 by staining smears of pus from the male urethra with methyl violet, and it was soon realised that it stained equally well with other basic aniline dyes such as gentian violet and methylene blue. Gram did not try his N technique on the gonococcus himself, but in 1886 Roux wrote: "One can always recognise the true nature [of gonococci] if, after establishing their pres- $\omega$ ence with gentian violet alone, the liquid of Gram and $\widetilde{\gamma}$ alcohol are successively added. If the cocci completely disappear, they are indeed those of Neisser." Counterstaining with Bismarck brown revealed the organisms more clearly, but later workers found that a better contrast was obtained with carbol fuchsin or methyl red.

Gram staining for the diagnosis of gonorrhoea was widely used by the end of the 19th century although some venereologists still preferred a single stain, particularly methylene blue. A few physicians-Harrison o was one-advocated staining by methylene blue to confirm the result of a Gram stain, but in modern times strict protocols have made such precautions unnecessary. Gram's is the commonest staining procedure in venereology, and indeed in the whole of clinical microbiology. In later life he was amused that he was best known for a single discovery that he had made, almost by chance, as a young man. He said he had been lucky, but luck often favours those who deserve it.

J D ORIEL 